KYUNGPOOK Math. J. 55(2015), 345-353

http://dx.doi.org/10.5666/KMJ.2015.55.2.345

pISSN 1225-6951 eISSN 0454-8124

(c) Kyungpook Mathematical Journal

\title{
Subnormality and Weighted Composition Operators on $L^{2}$ Spaces
}

\author{
Mohammad Reza Azimi \\ Department of Mathematics, Faculty of Sciences, University of Maragheh, Maragheh, \\ Iran \\ e-mail : mhr.azimi@maragheh.ac.ir
}

Abstract. Subnormality of bounded weighted composition operators on $L^{2}(\Sigma)$ of the form $W f=u f \circ T$, where $T$ is a nonsingular measurable transformation on the underlying space $X$ of a $\sigma$-finite measure space $(X, \Sigma, \mu)$ and $u$ is a weight function on $X$; is studied. The standard moment sequence characterizations of subnormality of weighted composition operators are given. It is shown that weighted composition operators are subnormal if and only if $\left\{J_{n}(x)\right\}_{n=0}^{+\infty}$ is a moment sequence for almost every $x \in X$, where $J_{n}=h_{n} E_{n}\left(|u|^{2}\right) \circ T^{-n}, h_{n}=d \mu \circ T^{-n} / d \mu$ and $E_{n}$ is the conditional expectation operator with respect to $T^{-n} \Sigma$.

\section{Introduction}

Weighted composition operators acting on certain function spaces are operators of the form $W f=u f \circ T$, where $T$ is a measurable transformation on a $\sigma$-finite measure space $(X, \Sigma, \mu)$ and $u$ is a weight function on $X$. These operators include composition operators of the form $C f=f \circ T$ and multiplication operators with symbol $u$. So far most general properties of composition operators and major classes of known normal, quasinormal, hyponormal and weak hyponormal composition operators have been widely studied by numerous mathematicians (See [1],[2],[13],[15],[17],[19]). Conditions for weighted composition operators to belong to some certain specific classes are found in [8]. However general properties of weighted composition operators have not been studied as well as composition operators. Originally subnormal composition operators have been studied by A. Lambert in [12] based on the standard moment sequence characterizations. Recently, in [18] subnormality of composition operators have been characterized in the Laplace density case. In this paper we shall use the standard moment sequence

Received March 00, 2014; revised May 00, 2014; accepted November 00, 2014.

2010 Mathematics Subject Classification: Primary 47B20; Secondary 47B38.

Key words and phrases: Subnormal, Weighted composition operators, Conditional expectation, Moment sequence. 
characterizations of subnormality of weighted composition operators.

\section{Preliminaries}

Let $(X, \Sigma, \mu)$ be a complete $\sigma$-finite measure space and $T$ be a nonsingular measurable transformation from $X$ onto $X$, i.e. $T^{-1} \Sigma \subseteq \Sigma$ and such that $\mu \circ T^{-1}$ is absolutely continuous with respect to $\mu$ which is denoted by $\mu \circ T^{-1} \ll \mu$. Given a real or complex valued measurable weight function $u$ on $X$, the weighted composition operator on $L^{2}(\Sigma):=L^{2}(X, \Sigma, \mu)$ induced by $T$ and $u$ is defined by $W f=u f \circ T$. It is worth reminding the reader that the nonsingularity of $T$ assures that $W$ is well defined as a mapping of equivalence classes of functions on the support of $u$. Let $h=h_{1}:=d \mu \circ T^{-1} / d \mu$ be the Radon-Nikodym derivative and for each $n \geq 1$ let $h_{n}:=d \mu \circ T^{-n} / d \mu$. For $f \in L^{2}(\Sigma)$ or $f \geq 0$ a.e let $E(f):=E\left(f \mid T^{-1} \Sigma\right)$ be the conditional expectation of $f$ with respect to $\sigma$-subalgebra $T^{-1} \Sigma$. For each $n \geq 2$, let $E_{n}(f):=E\left(f \mid T^{-n} \Sigma\right)$. Conditional expectation operator $E$ is defined as an orthogonal projection from $L^{2}(\Sigma)$ onto $L^{2}\left(T^{-1} \Sigma\right)$ by the assignment $f \rightarrow E(f)$. If $g$ is a $T^{-1} \Sigma$-measurable function then $E(f g)=g E(f)$. One may find more noteworthy properties of conditional expectation operator in $[6,16,2]$ and the references therein. In [15] it is shown that $C$ defines a bounded operator on $L^{2}(\Sigma)$ if and only if $h \in L^{\infty}(\Sigma):=L^{\infty}(X, \Sigma, \mu)$. Necessary and sufficient condition for $W$ to be bounded on $L^{2}(\Sigma)$ is that $J:=h E\left(|u|^{2}\right) \circ T^{-1} \in L^{\infty}(\Sigma)$ which is found in [7]. Conditional expectation is an functional projection and we shall use it in this paper frequently. For example observe the recurrent relationship $h_{n+1}=h E\left(h_{n}\right) \circ T^{-1}$ proved in [11].

A measurable transformation $T: X \rightarrow X$ is said to be essentially surjective if $\mu(X-T(X))=0$. It is shown in [9] that a bounded weighted composition operator $W$ is injective if and only if $T$ is essentially bounded. Throughout this paper it is assumed that $T$ is essentially bounded.

Recall that a bounded operator $A$ defined on a Hilbert space is normal if and only if $A^{*} A=A A^{*}$ (where $A^{*}$ is a adjoint of $A$ ) and $A$ is subnormal if $A$ has an extension to a normal operator on a larger Hilbert space. Let $A=U|A|$ be the canonical polar decomposition for $\mathrm{A}$ and let $p \in(0, \infty)$. An operator $A$ is p-hyponormal if $\left(A^{*} A\right)^{p} \geq\left(A A^{*}\right)^{p}$ and $\mathrm{A}$ is $p$-quasihyponormal if $A^{*}\left(A^{*} A\right)^{p} A \geq A^{*}\left(A A^{*}\right)^{p} A$. For all unit vectors $x \in \mathcal{H}$, if $\left\||A|^{p} U|A|^{p} x\right\| \geq\left\||A|^{p} x\right\|^{2}$, then $A$ is called a p-paranormal operator(See [5]). For instance consider the known relationships between these classed as follows

$$
\begin{gathered}
\text { normal } \Longrightarrow \text { quasinormal } \Longrightarrow \text { subnormal } \Longrightarrow \text { hyponormal }, \\
p \text { - hyponormal } \Longrightarrow p \text { - quasihyponormal } \Longrightarrow p \text { - paranormal. }
\end{gathered}
$$

Further information may be found in $[1,8,2]$.

\section{Subnormal Weighted Composition Operators}


The assumption that $T$ is essentially bounded; plays an important role to make use of the following theorem stated in [14] .

Theorem 3.1. Let $A$ be an operator on a Hilbert space $H$ with kernel $\{A\}=0$. Then $A$ is subnormal if and only if for every $x$ in $H$ the sequence $\left\{\left\|A^{n} x\right\|\right\}_{n=0}^{+\infty}$ is a moment sequence, i.e., there is an interval $I=[0, r]$ such that for each $x$ in $H$ one can find a Borel measure $m_{x}$ such that for each $n \geq 0$,

$$
\left\|A^{n} x\right\|^{2}=\int_{I} t^{n} d m_{x}(t) .
$$

Remark 3.2. For each $n \in \mathbb{N}$ and $F \in \Sigma$, define the measure $\lambda_{u}^{n}$ by

$$
\lambda_{u}^{n}(F)=\int_{T^{-n}(F)}|u|^{2} d \mu .
$$

According to the following chain

$$
\lambda_{u}^{n} \ll \mu \circ T^{-n} \ll \ldots \ll \mu \circ T^{-2} \ll \mu \circ T^{-1} \ll \mu,
$$

the assumption $\mu \circ T^{-1} \ll \mu$ implies that $\lambda_{u}^{n} \ll \mu$. Hence there exists the RadonNikodym derivative $J_{n}:=\frac{d \lambda_{u}^{n}}{d \mu}$. For $F \in \Sigma$, we have

$$
\begin{aligned}
\lambda_{u}^{n}(F) & =\int_{T^{-n}(F)}|u|^{2} d \mu \\
& =\int_{T^{-n}(F)} E_{n}\left(|u|^{2}\right) d \mu \\
& =\int_{F} h_{n} E_{n}\left(|u|^{2}\right) \circ T^{-n} d \mu .
\end{aligned}
$$

Then $d \lambda_{u}^{n}=h_{n}\left[E_{n}\left(|u|^{2}\right)\right] \circ T^{-n} d \mu$, since $F$ was chosen arbitrarily. It follows that $J_{n}=h_{n}\left[E_{n}\left(|u|^{2}\right)\right] \circ T^{-n}$. Note that $J_{1}=J$ and let $J_{0}=h_{0}=1$. Moreover, for an arbitrary $F \in \Sigma$,

$$
\begin{aligned}
\lambda_{u}^{n}(F) & =\int_{T^{-n}\left(T^{-1} F\right)}|u|^{2} d \mu \\
& =\int_{T^{-n}\left(T^{-1} F\right)} E_{n}\left(|u|^{2}\right) d \mu \\
& =\int_{T^{-1}(F)} h_{n} E_{n}\left(|u|^{2}\right) \circ T^{-n} d \mu \\
& =\int_{T^{-1}(F)} E\left(J_{n}\right) d \mu \\
& =\int_{F} h E\left(J_{n}\right) \circ T^{-1} d \mu .
\end{aligned}
$$


Then $J_{n+1}=h E\left(J_{n}\right) \circ T^{-1}$. If Theorem 3.1 is now applied to a bounded weighted composition operator we have the following.

Theorem 3.3. $W$ is subnormal if and only if for each $f \in L^{2}(\Sigma),\left\{\int_{x} J_{n}|f|^{2} d \mu\right\}_{n=0}^{+\infty}$ is a moment sequence.

Proof. Let $f \in L^{2}(\Sigma)$, by calculating the $n$-th iteration of $W$ we will have

$$
W^{n} f=\prod_{i=0}^{n-1}\left(u \circ T^{i}\right)\left(f \circ T^{n}\right) .
$$

Thus,

$$
\begin{aligned}
\left\|W^{n} f\right\|^{2} & =\int_{X}\left|\prod_{i=0}^{n-1}\left(u \circ T^{i}\right)\left(f \circ T^{n}\right)\right|^{2} d \mu \\
& =\int_{X} \prod_{i=0}^{n-2}\left|u \circ T^{i}\right|^{2}\left|f \circ T^{n-1}\right|^{2} d \lambda_{u}^{1} \\
& =\int_{X} \prod_{i=0}^{n-3}\left|u \circ T^{i}\right|^{2}\left|f \circ T^{n-2}\right|^{2} d \lambda_{u}^{2} \\
& \vdots \\
& =\int_{X}|f|^{2} d \lambda_{u}^{n} \\
& =\int_{X} J_{n}|f|^{2} d \mu .
\end{aligned}
$$

In what follows we are going to determine the subnormality of the weighted composition operators just by the sequence $\left\{J_{n}\right\}_{n=0}^{+\infty}$ based on the following standard moment sequence characterization.

Theorem 3.4. Let $\left\{\lambda_{n}\right\}_{n=0}^{+\infty}$ be a sequence of positive real numbers. Then $\left\{\lambda_{n}\right\}_{n=0}^{+\infty}$ is moment sequence if and only if for some interval $I$ the linear functional $\varphi$ defined on $P(I)$ - the set of polynomials over $I$ - by $\varphi\left(\sum_{n=0}^{+\infty} a_{n} t^{n}\right)=\sum_{n=0}^{+\infty} a_{n} \lambda_{n}$ is positive (i.e., $\varphi(p) \geq 0$ whenever $P(t) \geq 0$ on $I$ ).

Proof. See [20].

The application of above theorem characterizes subnormality of weighted composition operators initially.

Theorem 3.5. Let $W \in \mathcal{B}\left(L^{2}(\Sigma)\right)$ and $I=\left[0,\|J\|_{\infty}\right]$. Define the linear transformation $\mathcal{L}: P(I) \rightarrow L^{\infty}(\Sigma)$ by $\mathcal{L}\left(\sum_{n=0}^{+\infty} a_{n} t^{n}\right)=\sum_{n=0}^{+\infty} a_{n} J_{n}$. Then $W$ is subnormal if and only if $\mathcal{L}$ is positive, in a sense that $\mathcal{L}(p) \geq 0$ a.e $[\mu]$ whenever $p \geq 0$ on I. 
Proof. Let $W$ be a subnormal and $p$ be an arbitrary nonnegative polynomial on $I$. Let $K \subseteq\{x \in X: \mathcal{L}(p)(x)<0\}$ with $\mu(K)<+\infty$. By Theorem 3.3 there is a Borel measure $m$ on $I$ such that for every $n \geq 0$,

$$
\int_{K} J_{n} d \mu=\int_{I} t^{n} d m(t)
$$

Then

$$
\begin{aligned}
\int_{K} \mathcal{L}(p) d \mu & =\sum_{n=0}^{+\infty} a_{n} \int_{K} J_{n} d \mu \\
& =\sum_{n=0}^{+\infty} a_{n} \int_{I} t^{n} d m(t) \\
& =\int_{I} p(t) d m(t) \geq 0 .
\end{aligned}
$$

It follows that $\mathcal{L}(p) \geq 0$ a.e, hence $\mathcal{L}$ is positive.

Conversely, suppose that $\mathcal{L}$ is positive. Note that $\mathcal{L}$ is continuous with respect to essential sup norm since $\mathcal{L}$ preserves the unit element of both sides and $J_{n}(x) \in$ $I=\left[0,\|J\|_{\infty}\right]$ a.e for each $n \geq 0$. Then $\mathcal{L}$ can be normally extended to a positive linear mapping from $C(I)$ (the space of all continuous functions on $I$ ) into $L^{\infty}(\Sigma)$. Correspond for each $f \in L^{2}(\Sigma)$ a linear functional $\varphi_{f}$ on $C(I)$ by

$$
\varphi_{f}(g)=\int_{X} \mathcal{L}(g)|f|^{2} d \mu
$$

Since $\mathcal{L}(g) \in L^{\infty}(\Sigma), \varphi_{f}$ is bounded. Actually its norm can not be exceed $\|\mathcal{L}\|\|f\|_{2}^{2}$. By the Riesz representation theorem there is a unique Borel measure $m_{f}$ such that

$$
\varphi_{f}(g)=\int_{I} g(t) d m_{f}(t), \quad g \in C(I)
$$

Hence $\int_{X} \mathcal{L}(g)|f|^{2} d \mu=\int_{I} g(t) d m_{f}(t)$. For each $n \geq 0$, putting $g(t)=t^{n}$ in the last equation leads that

$$
\int_{X} J_{n}|f|^{2} d \mu=\int_{I} t^{n} d m_{f}(t)
$$

It follows from Theorem 3.3 that $W$ is subnormal.

Corollary 3.6. $W \in \mathcal{B}\left(L^{2}(\Sigma)\right)$ is subnormal if and only if for every $\Sigma$-measurable set $A$ of finite measure the sequence $\left\{\int_{T^{-n} A}|u|^{2} d \mu\right\}_{n=0}^{+\infty}$ is a moment sequence. 
Proof. First suppose that $W$ is subnormal. Then by Theorem 3.3 we have

$$
\begin{aligned}
\int_{X} J_{n} \chi_{A} d \mu & =\int_{A} h_{n}\left[E_{n}\left(|u|^{2}\right)\right] \circ T^{-n} d \mu \\
& =\int_{T^{-n} A} E_{n}\left(|u|^{2}\right) d \mu \\
& =\int_{T^{-n} A}|u|^{2} d \mu .
\end{aligned}
$$

Conversely, let $A$ be an arbitrary $\Sigma$-measurable set of finite measure on which $\left\{\int_{T^{-n} A}|u|^{2} d \mu\right\}_{n=0}^{+\infty}$ is a moment sequence. Let $p(t) \geq 0$ on $I=\left[0,\|J\|_{\infty}\right]$. Then there is a Borel measure $m$ such that for each $n, \int_{T^{-n} A}|u|^{2} d \mu=\int_{I} t^{n} d m(t)$. Then

$$
\begin{aligned}
\int_{A} \mathcal{L}(p) d \mu & =\sum_{n=0}^{+\infty} a_{n} \int_{A} J_{n} d \mu \\
& =\sum_{n=0}^{+\infty} a_{n} \int_{T^{-n} A}|u|^{2} d \mu \\
& =\sum_{n=0}^{+\infty} a_{n} \int_{I} t^{n} d m(t) \\
& =\int_{I} p(t) d m(t) \\
& \geq 0 .
\end{aligned}
$$

Hence $\mathcal{L}(p) \geq 0$ a.e, since $A$ was chosen arbitrarily. This fact yields that $W$ is subnormal by Theorem 3.5.

Proposition 3.7. $W \in \mathcal{B}\left(L^{2}(\Sigma)\right)$ is subnormal if and only if $\left\{J_{n}(x)\right\}_{n=0}^{+\infty}$ is a moment sequence for almost every $x \in X$.

Proof. The proof follows very closely the proof of Corollary 4 in [12]. Suppose first that $W$ is subnormal. Let $P^{+}(I)$ be the set of all nonnegative polynomials on $I$. Let $P_{r}^{+}(I)$ be the set of all polynomials with rational coefficients which are nonnegative on $I$. By Theorem 3.5 for each $p \in P_{r}^{+}(I), \mathcal{L}(p) \geq 0$ a.e. Let $X_{p}:=\{x \in X: \mathcal{L}(p) \geq 0\}$. Then $\mu\left(X-X_{p}\right)=0$. Since $P_{r}^{+}(I)$ is a countable dense subset of $P^{+}(I)$ we have $\mu\left(X-X^{*}\right)=0$ where $X^{*}=\bigcup\left\{X_{p}: p \in P_{r}^{+}(I)\right\}$. It means that for each $p \in P^{+}(I), \mathcal{L}(p) \geq 0$ a.e. Therefore by Theorem 3.5 it is inferred that $\left\{J_{n}(x)\right\}_{n=0}^{+\infty}$ is a moment sequence for every $x \in X^{*}$.

Conversely, suppose that $\left\{J_{n}(x)\right\}_{n=0}^{+\infty}$ is a moment sequence for almost every $x \in X$. Let $X_{0}$ be a measurable subset of $X$ with $\mu\left(X-X_{0}\right)=0$ in a way that $\left\{J_{n}(x)\right\}_{n=0}^{+\infty}$ is a moment sequence on $X_{0}$. If $p$ is an arbitrary nonnegative polynomial on $I$, then by Theorem 3.4, $\mathcal{L}(p) \geq 0$ exactly on $X_{0}$. Thus the positivity of $\mathcal{L}$ yields that $W$ is subnormal by Theorem 3.5.

Example 3.8. Consider the case that $h \circ T=h$ and $E\left(|u|^{2}\right) \circ T=E\left(|u|^{2}\right)$. This case in turn characterizes $p$-quasihyponormal and $p$-paranormal weighted composition 
operators; see [8]. Now the relationship $J_{n+1}=h E\left(J_{n}\right) \circ T^{-1}$ follows that $J_{n}=$ $h^{n-1} J$ for each $n \geq 0$. Thus $\mathcal{L}\left(\sum_{n=0}^{+\infty} a_{n} t^{n}\right)=a_{0}+J \sum_{n=1}^{+\infty} a_{n} h^{n-1}$ is positive since $J(x) \in\left[0,\|J\|_{\infty}\right]$ for almost every $x \in X$. Therefore $W$ is subnormal by Theorem 3.5 .

Example 3.9. Let $X$ be the set of nonnegative integers, let $\Sigma$ be the $\sigma$-algebra of all subsets of $X$, and take $\mu$ to be the point mass measure determined by the $\mu(k):=m_{k}, \quad k \in X$. Define the measurable transformation $T$ by

$$
T(k)= \begin{cases}0 & k=0,1 \\ k-1 & k \geq 2\end{cases}
$$

and the weight function $u$ by

$$
u(k)= \begin{cases}1 & k=0,1 \\ k & k \geq 2\end{cases}
$$

By routine computations one may verify that

$$
\begin{aligned}
h(k) & =\frac{1}{m_{k}} \sum_{j \in T^{-1}(k)} m_{j} \\
h_{n}(k) & =\frac{1}{m_{k}} \sum_{j \in T^{-1}(k)} h_{n-1}(j) m_{j} \\
J_{n}(k) & =\frac{1}{m_{k}} \sum_{j \in T^{-n}(k)}(u(j))^{2} m_{j} .
\end{aligned}
$$

So by inserting $T$ and $u$ in the above formulas we obtain

$$
\begin{aligned}
J_{1}(0) & =\frac{1}{m_{0}}\left(m_{0}+m_{1}\right) ; \\
J_{n}(0) & =\frac{1}{m_{0}}\left(m_{0}+m_{1}+2^{2} m_{2}+3^{2} m_{3}+\ldots+n^{2} m_{n}\right) ; \\
J_{n}(k) & =\frac{m_{k+n}}{m_{k}}, \quad k \geq 1 .
\end{aligned}
$$

In particular, fix $a>0$ and let $\epsilon>0$ be an arbitrary. Let $m_{0}=\epsilon$ and for every $k \geq 1$, let

$$
m_{k}=\frac{1}{k^{2}} \int_{a}^{a+\epsilon}\left(t^{k}-t^{k-1}\right) d t .
$$

In Proposition 3.7 it is proved that $W$ is subnormal if and only if for every $k \geq 1$ the sequence $\left\{J_{n}(k)\right\}_{n=0}^{+\infty}$ is a moment sequence. Namely the sequences $\left\{m_{0}+\right.$ $\left.\sum_{i=1}^{n} i^{2} m_{i}\right\}_{n=0}^{+\infty}$ and $\left\{\frac{m_{k}+n}{m_{k}}\right\}_{n=0}^{+\infty}$ are moment sequences. But these assertions are 
true since for every $k \geq 1$ and $n \geq 0$, we have

$$
\begin{aligned}
m_{0}+\sum_{i=1}^{n} i^{2} m_{i} & =m_{0}+\sum_{i=1}^{n} \int_{a}^{a+\epsilon}\left(t^{i}-t^{i-1}\right) d t \\
& =\int_{a}^{a+\epsilon} t^{n} d t
\end{aligned}
$$

and

$$
\begin{aligned}
\frac{m_{k+n}}{m_{k}} & =\frac{1}{(k+n)^{2} m_{k}} \int_{a}^{a+\epsilon}\left(t^{k+n}-t^{k+n-1}\right) d t \\
& =\int_{a}^{a+\epsilon} t^{n}\left(\frac{t^{k}-t^{k-1}}{(k+n)^{2} m_{k}}\right) d t \\
& =\int_{a}^{a+\epsilon} t^{n} d \mu(t),
\end{aligned}
$$

where $d \mu=\left(\frac{t^{k}-t^{k-1}}{(k+n)^{2} m_{k}}\right) d t$ is a nonnegative measure on $[a, a+\epsilon]$. Hence $W$ is subnormal.

\section{References}

[1] C. Burnap, I. Jung, Composition operators with weak hyponormality, J. Math. Anal. Appl., 337(2008), 686-694.

[2] C. Burnap, I. Jung and A. Lambert, Separating partial normality classes with composition operators, J. Operator Theory, 53(2005), 381-397.

[3] J. B. Conway, Subnormal Operators, Pitman Publ. Co., London, 1981.

[4] J. B. Conway, The Theory of Subnormal Operators, Math. Surveys Monogr., Amer. Math. Soc., Providence, RI, 1991.

[5] M. Fujii, S. Izumino and R. Nakamoto, Classes of operators determined by the HeinzKato-Furuta inequality and the Hölder- McCarthy inequality, Nihonkai Math. J., 5(1994), 61-67.

[6] J. Herron, Weighted conditional expectation operators, Oper. Matrices, 5(2011), 107118.

[7] T. Hoover, A. Lambert and J. Quinn, The Markov process determined by a weighted composition operator, Studia Math., 72(3)(1982), 225-235.

[8] M. R. Jabbarzadeh, M. R. Azimi, Some weak hyponormal classes of weighted composition operators, Bull. Korean Math. Soc., 47(4)(2010), 793-803.

[9] R. Kumar, Ascent and descent weighted composition operators on $L^{p}$-spaces, Math. Vesnik, 60(2008), 47-51. 
[10] A. Lambert, Thomas G. Lucas, Nagata's principle of idealization in relation to module homomorphisms and conditional expectations, Kyungpook Math. J., 40(2000), 327337.

[11] A. Lambert, Normal extentions of subnormal compostion operators, Michigan Math. J., 35(1988), 443-450.

[12] A. Lambert, Subnormal composition operators, Proc. Amer. Math. Soc., 103(1988), 750-754.

[13] A. Lambert, Hyponormal composition operators, Bull. Lond. Math. Soc., 18(1986), 395-400.

[14] A. Lambert, Subnormality and weighted shifts, Bull. Lond. Math. Soc., 14(2)(1976), 476-480.

[15] E. Nordgren, Composition operators on Hilbert spaces, in: Lecture Notes in Math., 693, Springer-Verlag, Berlin, 1978.

[16] M. M. Rao, Conditional measure and applications, Marcel Dekker, New York, 1993.

[17] R. K. Singh, J. S. Manhas, Composition Operators on Function Spaces, Elsevier Science Publishers B. V., North-Holland, Amsterdam, 1993.

[18] J. Stochel, J. B. Stochel, Seminormal composition operators on L2 spaces induced by matrices: The Laplace density case, J. Math. Anal. Appl., 375(2011), 1-7.

[19] R. Whitley, Normal and quasinormal composition operators, Proc. Amer. Math. Soc., 70(1978), 114-118.

[20] D. Widder, The Laplace transform, Princeton Univ. Press, Princeton, N. J., 1946. 